\title{
Interpreting Facial Expression: A Challenging Study Using Existing Video
}

\author{
Ira Darmawanti \\ Department of Education \\ University of Vienna \\ Vienna, Austria \\ irad62@univie.ac.at
}

\begin{abstract}
The study aims to investigate the challenging situation that might be faced when using an existing video to interpret facial expressions within the context of a classroom situation. Facial expressions, as part of non-verbal communication, are a crucial means of sending messages between the teacher and students. Most studies assess facial expressions using static rather than moving images. One way to examine facial expressions deriving from the interactions between teacher and students, which are always represented as moving images, can be carried out using existing video. This study utilizes literature to investigate the challenges in assessing facial expression using existing video and will contribute to the scope of knowledge on this topic.
\end{abstract}

Keywords-interpreting; facial expressions; existing video; study literature

\section{INTRODUCTION}

Facial expression is crucial to the exchange of messages when communicating with others, and therefore also in the non-verbal communication between teachers and students. Facial expressions are commonly used to identify specific states, such as sleepiness, relaxation, confusion, boredom, interest, and attention [1], and may be effectively used by teachers as feedback, adjusting their teaching style accordingly, speeding up or slowing down [2]. From the students' point of view, facial expression may help to understand the teacher's states of mind, like anger or a smile, allowing students to change their behaviour to foster learning in class [3]. All of these can be a crucial point in the learning and teaching process.

Facial expression is closely related to emotion [1], and it consistently reflects between emotions and experiences [4]. Specific signals on the face portray different emotions; for example, a raised brow often denotes happiness [5].

On the one hand, facial expression may be incorrectly interpreted by teachers or students because of a number of factors: firstly, when it is paired with body language, the interpretation of facial expression may have the opposite meaning. In addition, what is observed and uttered by people simultaneously is often contradictory. For example, the teacher sometimes indicates verbal agreement with her student, but at the same time, she turns away and shrugs her shoulders, which may send the opposite message. Another example would be a student who winks at his neighbours while answering a question from his teacher: this could represent doubt or have a negative connotation [6]. Secondly, there is a condition in which observers are not able to correctly interpret the emotion in facial expression: displaying an angry expression on a face, therefore, does not necessarily imply that it will be recognized as such by the observer [7]. All these situations may lead to misunderstandings between the teacher and students because of differences in interpretation. Because of the reasons listed above, facial expression requires further investigation.

Current studies on facial expression indicate how the debate as to whether facial expression is universal, e.g., [8], [9] or cultural, e.g., [10], [11] still persists. Some studies discuss the five categories of emotions into which facial expression can be divided: anger, fear, enjoyment, sadness, and disgust [12]. Another study identifies seven categories of facial expression: happiness, surprise, fear, anger, contempt, disgust, and sadness Still others state that there are six different emotions, which are happiness, surprise, fear, anger, disgust, and sadness [13], [14].

Despite these differences, what all studies have in common is the study attempts to examine facial expression based on cultural background; some researchers recruit participants from different cultures [5], [15], [16]. Furthermore, as illustrated in the examples above, the quantitative method is often applied in measuring facial expression across culture. Finally, facial expression is interpreted by using a static image [8], [10], [11].

One further way to assess facial expression in the classroom situation is to use video, since video furnishes detail and accurate data as it can be viewed and analysed repeatedly [17], [18]. However, most of the studies assess facial expression using a static image, see [8], [10], [11], instead of a video that provides a moving image. Actually, facial expression resulting from a classroom interaction can be more effectively recognized in a moving image".

Most studies relating to classroom situations seem to focus on producing videos [19], which serve as an observational tool for teachers, who can reflect on their teaching skills within the classroom setting [20], [21], [22]. Video has become a 
common tool in teachers' education, and is used in many institutions today.

When using existing video, the data that it contains portraying the classroom situation can be reviewed and reanalysed. Since the video has many 'lenses' [23], it can be readjusted in order to be examined from different perspectives. In other words, the aim of the study might be different from the video's original purpose. Here, the video functions as a research tool [24], or rather, as a source of potential data [25].

Therefore, as potential data, it would appear advantageous to examine facial expressions within the classroom situation using existing video. However, the study using existing video in the educational field is still in its early phases and has yet to be developed [24]. It is therefore the aim of this present paper to attempt to investigate it and answer the question: What are the challenges that might arise when interpreting facial expressions using the existing video of a classroom situation?

\section{METHOD}

By studying the available literature, this paper will provide a better understanding regarding the assessment of facial expressions using existing video in a classroom setting.

\section{RESULTS AND DISCUSSION}

The complexity uses existing video in interpreting facial expression might lead some potential problems that can be illustrated as follows.

\section{A. Instruction}

The complexity of data analysis using produced video springs from the training necessary in order to correctly manage several devices at once [26]. Specific guidance will be necessary in how to correctly arrange devices in the field, as well as how to control them [27]. The complexity of using existing video, on the other hand, lies in the instruction (guidance) in selecting for interpretation those segments which are most representative of facial expressions in the classroom situation. Here, clear instruction is essential when using existing video and constitutes the preliminary phase before interpretation is possible. If the camera position is crucial when video is produced for the study [17], [27], when existing video is utilized, it is the selected segment based on camera perspective which is most important. The distance and speed of the camera when shooting (zooming) the actors' faces (teacher or students) in class should be considered. Here, some questions may arise [27]: is the existing video shot using a fixed or mobile camera? What is the distance between the camera and the actor(s)? How much time is needed (to interpret facial expression) when the camera is shooting an actor's face? Is there any standardized distance and time (to interpret the facial expression)? Taking all these factors into account will facilitate the selection of segments for analysis.

\section{B. The Challenge of Technology}

When interpreting facial expressions using existing video, selecting high-quality video is necessary [17]. If the quality of the video recording is inferior, and cutting-edge technology has not been used, this problem can usually be solved as long as the equipment is compatible. Even though it seems that the development of technology has not played a major role in the effectiveness of analysis [28], changing technology has an impact in collecting, constructing, analyzing, and interpreting the data [18]. Therefore, adjusting to changing technology is indispensable.

\section{The Data Produced}

It is obvious that the data produced in video show a greater range of visual, kinesthetic, verbal, and gestural, expressions, as well as furnishing details pertaining to the environment [26]. Since the video contains abundant data, only a limited amount of information captured by the video camera should be observed in order to be interpreted [22]. In other words, only a selection of relevant data will be studied. This might also be applicable to studies which use existing video and which contain a great deal of data that must undergo a selection process.

Unlike using static images (photos), which are not influenced by sound (intonation), language, or gestures, when moving images are utilized the intonation, language or gestures may have an impact on the study [23]. This might also occur if the aim of the study is to examine facial expressions using existing video. Some questions thus arise: how can facial expressions be interpreted as a result of the interaction? Can they be interpreted independently or do they occur simultaneously with gestures, language, or intonation? How is any differentiation between these factors possible?

Furthermore, the data produced is associated with naturalness. The data regarding the degree of naturalness obtainable refer to the presence of the researcher while recording the situation and affecting the actors [29] as little as possible. Here, the actor filmed in the video behaves as if they were not being observed. The presence of the researcher or the video equipment may exert some impact on the situation being recorded: this is also referred to as 'reactivity' [30], [31]. If this occurs, it raises other questions when using existing video: how can facial expression be recognised as natural? Did the expression occur because of reactivity and not naturally? How can naturally be distinguished from reactivity when examining facial expression?

A further challenging situation might arise if the study focuses on facial expression coupled with speech (verbal); in this case, both elements must be assessed concurrently. Here, even though the video data can be analysed repeatedly [17], [18], it must be kept in mind that playing video frequently and in slow motion may alter the sound quality [26]. This might influence the analysis.

\section{Analysis and Interpretation}

The data produced must be transcribed during the first phase of analysis, as it possesses crucial importance for further stages of analysis. In addition, there should be a match between transcription and images [18]. If the study intends to 
use verbal (speech), which sometimes requires more than one camera or recorder, the transcription and analysis must be based on a combination of precise images and clear speech. The fact that facial expressions sometimes occur alongside speech and gestures or gazes gives rise to other queries, such as how the researcher is able to transcribe gestures or gazes and combine these with speech so that these transcriptions can be read and easily understood by the scholar [32].

\section{E. Ethical Issues}

Conducting research using video produced for this purpose as well as video analysis using existing video raises some ethical issues. Discussion of these ethical issues may be addressed as follows [17], [18], [33]. The first question which needs to be addressed is that of filming permits, who is allowed to record, which parts and segments of the images may be stored, analysed, or published. Second, and closely related to the first, is to guarantee the informed consent and anonymisation of moving images. Third, is the right to privacy. All of these issues remain unsolved since they pertain to the legal systems in terms of data protection. Thus, the researcher is confronted with the dilemma of protecting the rights of the individual being filmed while still guaranteeing the freedom of research.

\section{CONCLUSION}

To conclude, as illustrated above, questions and challenges for future research remain to be addressed in the study on interpreting facial expression using existing video of a classroom situation. Attention to detail and precise steps are essential in conducting the study. The discussion above offers its contribution towards gaining a better understanding of the interpretation of facial expression using existing video within the context of a classroom situation. In other words, it can be useful as a tool of reflection when evaluating the fundamental steps for future research.

\section{ACKNOWLEDGMENT}

The author is grateful for the funding by the Ministry of Research, Technology and Higher Education of the Republic of Indonesia as well as the OeAD (Österreichische Austauschdienst-GmbH) in the Indonesia Austria Scholarship Program (IASP).

\section{REFERENCES}

[1] J. Russell, "Is there universal recognition of emotion from facial expression? A review of the cross-cultural studies," Psychological Bulletin, vol. 115, no. 1, pp. 102-141, 1994.

[2] M. Sathik and S. G. Jonathan, "Effect of facial expressions on student's comprehension recognition in virtual educational environments," Springerplus, vol. 2, no. 1, p. 455, 2013.

[3] M. N. Butt and M. Iqbal, "Teachers' perception regarding facial expressions as an effective teaching tool," Contemporary Issues in Education Research, vol. 4, no. 2, pp. 11-14, 2011.

[4] D. Keltner, "Ekman, emotional expression, and the art of empirical epiphany," Journal of Research in Personality, vol. 38, no. 1, pp. 37 44, 2004.

[5] C. F. Keating et al., "Culture and the perception of social dominance from facial expression.," Journal of Personality and Social Psychology, vol. 40, no. 4, pp. 615-626, 1981, vol. 40, no. 4, p. 615, 1981.

[6] J. Engel and B. Fritzsche, "The potential of videography in comparative education: Introduction." SAGE Publications Sage UK: London, England, 2015.

[7] J. A. Russell and J. M. Fernández-Dols, "What does a facial expression mean?," The Psychology of Facial Expression, J. A. Russell and J. M. Fernández-Dols, Eds., Cambridge: Cambridge University Press, 1997. pp. 3-30.

[8] H. A. Elfenbein and N. Ambady, "On the universality and cultural specificity of emotion recognition: a meta-analysis.," Psychological. Bulletin., vol. 128, no. 2, p. 203, 2002.

[9] J. Haidt and D. Keltner, "Culture and facial expression: Open-ended methods find more expressions and a gradient of recognition," Cognition and Emotion., vol. 13, no. 3, pp. 225-266, 1999.

[10] T. Shioiri, T. Someya, D. Helmeste, and S. W. Tang, "Misinterpretation of facial expression: A cross- cultural study," Psychiatry and Clinical Neurosciences, vol. 53, no. 1, pp. 45-50, 1999.

[11] Y. Huang, S. Tang, D. Helmeste, T. Shioiri, and T. Someya, "Differential judgement of static facial expressions of emotions in three cultures," Psychiatry and Clinical Neurosciences., vol. 55, no. 5, pp. 479-483, 2001.

[12] P. Ekman, "An argument for basic emotions," Cogn. Emot., vol. 6, no. 3-4, pp. 169-200, 1992.

[13] M. N. Carminati and P. Knoeferle, "Effects of speaker emotional facial expression and listener age on incremental sentence processing," PLoS One, vol. 8, no. 9, p. e72559, 2013.

[14] S.-M. Hsu and L.-X. Yang, "Sequential effects in facial expression categorization.," Emotion, vol. 13, no. 3, p. 573, 2013.

[15] K. Koelkebeck et al., "Benefits of using culturally unfamiliar stimuli in ambiguous emotion identification: A Cross-cultural study," Psychiatry Research, vol. 228, no. 1, pp. 39-45, 2015.

[16] T. Koda, T. Ishida, M. Rehm, and E. André, "Avatar culture: crosscultural evaluations of avatar facial expressions," AI Soc., vol. 24, no. 3, pp. 237-250, 2009.

[17] C. Heath, J. Hindmarsh, and P. Luff, Video in qualitative research. Analysing social interaction in everyday life. London: SAGE, 2010

[18] H. Knoblauch, B. Schnettler, and J. Raab, "Video-analysis, methodological aspect of interpretative audiovisual analysis in social research," in Video-Analysis Methodology and Method: Qualitative Audiovisual Data Analysis in Sociology, H. Knoblauch, B. Schnettler, J. Raab, and H. G. Soeffner Eds., 3rd Revised Editon ed. Frankurt: Peter Lang GmbH, 2012.

[19] E. Susantini, U. Faizah, M. S. Prastiwi, and Suryanti, "Developing educational video to improve the use of scientific approach in cooperative learning," Journal of Baltic Science Education, vol. 15, no. 2, pp. 725-737, 2016

[20] T. Abodeeb-Gentile and R. Greenfield, "The use of video feedback in teacher education," in ICET 2016. 60th World Assembly. Teachers for a Better World: Creating Conditions for Quality Education-Pedagogy, Policy and Professionalism, The University of West Indies. Kingston, Jamaica, C. H. Getles, Ed., July 18-21 2016, Mona, Kingston, 2017

[21] M. A. Sherin and E. A. Van Es, "Using video to support teachers' ability to notice classroom interactions," Journal of Technology and Teacher Education, vol. 13, no. 3, pp. 475-491, 2005.

[22] U. Töman, "Investigation to improve the process of pre-service teachers' reflective thinking skills through an action research," Universal Journal of Educational Research, vol. 5, no. 9, pp. 1535-1548

[23] M. Gamoran Sherin, "New perspectives on the role of video in teacher education," in Using video in teacher education, Emerald Group Publishing Limited, 2003, pp. 1-27.

[24] H. Schluß and M. Jehle, Videodokumentation von Unterricht: Zugänge 
zu einer neuen Quellengattung der Unterrichtsforschung. Wiesbaden: Springer VS, 2013.

[25] F. Erickson, "Uses of video in social research: A brief history.," International Journal of Social Research Methodology, 2011.

[26] H. Knoblauch, R. Tuma, and B. Schnettler, Videography. Introduction to interpretative videoanalysis of social situations. Frankfurt: Peter Lang $\mathrm{GmbH}, 2015$.

[27] C. Jewitt, “An introduction to using video for research,” 2012.

[28] E. De Freitas, "The moving image in education research: Reassembling the body in classroom video data," International Journal of Qualitative Studies, vol. 29, no. 4, pp. 535-572, 2016.

[29] D. Silverman, "Instances or sequences? Improving the state of the art of qualitative research," in Forum Qualitative Sozialforschung/Forum:
Qualitative Social Research, vol. 6, no. 3, 2005.

[30] E. Laurier and C. Philo, "Natural problems of naturalistic video data," Video-analysis Methodol. methods, Qual. Audiov. data Anal. Sociol., pp. 183-192, 2006

[31] H. Lomax and N. Casey, "Recording social life: Reflexivity and video methodology," Sociological Research Online, vol. 3, no. 2, pp. 1-26, 1998.

[32] J. Bezemer and D. Mavers, "Multimodal transcription as academic practice: a social semiotic perspective," International Journal of Social Research Methodology, vol. 14, no. 3, pp. 191-206, 2011.

[33] B. Schnettler and J. Raab, "Interpretative visual analysis developments, state of the art and pending problems," Forum: Qualitative social research. Sozialforschung, vol. 9, no. 3, 2008. 